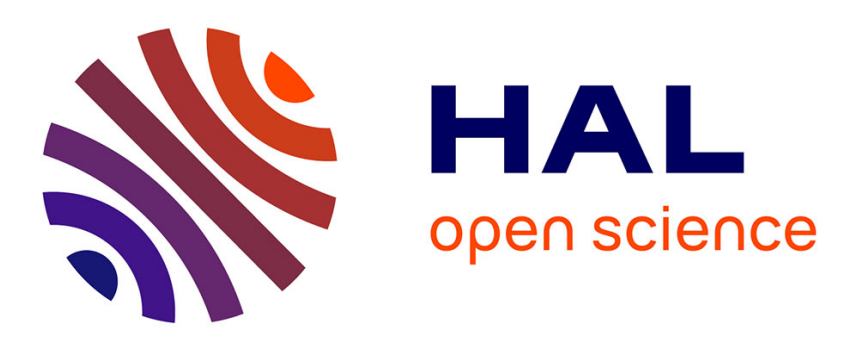

\title{
Highly Sensitive Miniaturized Refractive Index Sensor Based on Au-Ag Surface Gratings on a Planar Optical Waveguide
}

Saurabh Mani Tripathi, Arun Kumar, Emmanuel Marin, Jean-Pierre Meunier

\section{- To cite this version:}

Saurabh Mani Tripathi, Arun Kumar, Emmanuel Marin, Jean-Pierre Meunier. Highly Sensitive Miniaturized Refractive Index Sensor Based on Au-Ag Surface Gratings on a Planar Optical Waveguide. Journal of Lightwave Technology, 2010, 28 (17), pp.2469-2476. hal-00549352

\section{HAL Id: hal-00549352 https://hal.science/hal-00549352}

Submitted on 21 Dec 2010

HAL is a multi-disciplinary open access archive for the deposit and dissemination of scientific research documents, whether they are published or not. The documents may come from teaching and research institutions in France or abroad, or from public or private research centers.
L'archive ouverte pluridisciplinaire HAL, est destinée au dépôt et à la diffusion de documents scientifiques de niveau recherche, publiés ou non, émanant des établissements d'enseignement et de recherche français ou étrangers, des laboratoires publics ou privés. 


\title{
Highly Sensitive Miniaturized Refractive Index Sensor Based on Au-Ag Surface Gratings on a Planar Optical Waveguide
}

\author{
Saurabh Mani Tripathi, Member, IEEE, OSA, Arun Kumar, Member, IEEE, OSA, \\ Emmanuel Marin, Member, IEEE, OSA, and Jean-Pierre Meunier, Member, IEEE, OSA
}

\begin{abstract}
We present the theoretical study of a novel highly sensitive, miniaturized, integrated optic refractive index sensor based on a Au-Ag surface grating. The grating is considered to be made of alternate layers of equi-thick $\mathrm{Au}$ and $\mathrm{Ag}$ regions along the direction of propagation, on the surface of the waveguide. Due to the same thickness of both the metals, the surface plasmon polaritons (SPP) for both metals have their field maxima at the same transverse distance, leading to an increased modal overlap in the grating region and hence a reduced grating length. An exact coupled-mode-theory based on the local mode matching has been used to analyze the mode coupling between the guided mode and the SPP. It has been shown that the proposed design requires nearly one fourth of the grating length as compared to the corrugated metal grating for the same metal thickness. Further, for co-propagating mode coupling (LPG based sensor) the structure is found to be maximum sensitive at an optimum metal thickness, however, such an optimum metal thickness does not exist for counter-propagating coupling (FBG based sensor).
\end{abstract}

Index Terms-Integrated optic waveguides, refractive index sensor, surface gratings, surface plasmon polariton.

\section{INTRODUCTION}

I $\mathrm{N}$ the recent past there has been a growing interest in exciting the surface plasmon polaritons (SPPs) using optical waveguide gratings for their possible applications in biochemical sensing. Several structures based on the power coupling between counter propagating as well as co-propagating guided and SPP modes have been studied [1]-[9]. In most of these structures the grating is assumed to be written in the waveguide core region (higher index photosensitive region). Such gratings, however, are relatively weak in nature and since the modal overlap between the modes is very small, due to the sharply decaying SPP field beyond metal/dielectric interface, the required grating length to couple an appreciable amount of power is very large (typically ranging to several centimeters)

Manuscript received March 11, 2010; revised May 12, 2010; accepted May 25, 2010. Date of publication June 14, 2010; date of current version August 16, 2010.

S. M. Tripathi and A. Kumar are with the Physics Department, Indian Institute of Technology Delhi, New Delhi, Hauz Khas 110016, India (e-mail: tripathi. iit@gmail.com; akumar@physics.iitd.ernet.in)

E. Marin and J. P. Meunier are with the Universite de Lyon, F-42023 Saint-Etienne, France, and also with CNRS, UMR 5516, Laboratoire Hubert Curien, F-42000 Saint-Etienne, France, and also with the Université de Saint-Etienne, Jean-Monnet, F-42000 Saint-Etienne, France. (e-mail: emmanuel.marin@univ-st-etienne.fr; meunier@univ-st-etienne.fr)

Digital Object Identifier 10.1109/JLT.2010.2051796
[1]-[8]. For device miniaturization and ease of fabrication, however, a shorter grating length is preferred, which is further useful in realizing the whole of the optical assembly on a single chip for the so called lab-on-a-chip (LOC) applications [10]-[14]. The modal overlap within the grating region can be increased by reducing the cladding thickness, which, however, increases the mode loss associated with the core mode and hence has limited scope in reducing the grating length. To increase the modal coupling within the grating region it is pertinent to write the grating in the metal region itself where the SPP has its field maxima. For example, Nemova and Kashyap have reported a refractive index sensor based on the corrugated metal grating assisted power coupling between core mode and the SPP [6], which is benefited from the large field present in the metal region. It has been reported [6] that even for a very thick cladding region $(\sim 4.5 \mu \mathrm{m})$ the grating length ranges typically between 4-6 cm, as compared to the typical grating lengths of $\sim 15-20$ $\mathrm{cm}$ for gratings written in the photosensitive core region even with thinner claddings $(\sim 1 \mu \mathrm{m})$. Lu and Huang [15] have recently studied the effect of mode loss on the performance of such sensors and have shown that such sensors would work only if the grating length is less than the propagation length of the SPP. Thus, a smaller grating length should always be preferred. In the case of corrugated metal gratings, the SPP maxima in consecutive regions do not coincide, resulting in a lower modal overlap at the corrugated junction, which can be increased by reducing the corrugation depth. However, this weakens the grating due to the reduced refractive index modulation, and therefore a compromise has to be made between the modal overlap and the refractive index modulation.

In order to reduce the grating length by maximizing the modal overlap within the grating region, we propose the grating to be made of equi-thick, alternate regions of two different metals. Such a structure can be realized by using the photolithography and liftoff techniques used in the fabrication of optical integrated circuits [16]. The refractive index difference between the two metals provides the necessary perturbation for power coupling between the core mode and the SPP and the same thickness of the metal regions facilitates the increased modal overlap. In this paper we have analyzed such a grating and have presented its ambient refractive index sensing characteristics both in the co-propagating and counter-propagating regimes. Modal coupling from the core mode to both, the co-propagating SPP as well as counter-propagating SPP has been studied. The structure of the paper is as follows. In Section II, the sensor design and the mode propagation characteristics have been discussed. In Section III, an analysis of mode coupling, based on 

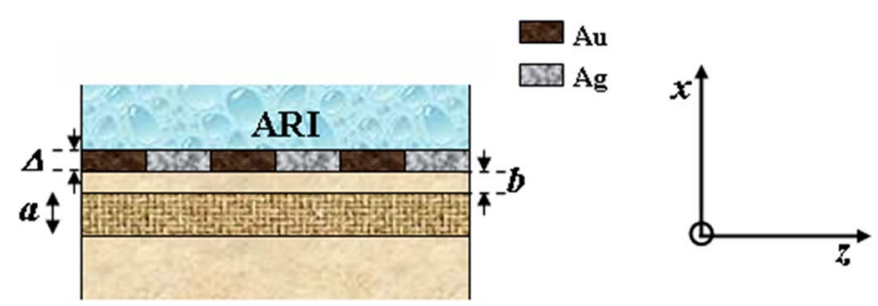

Fig. 1. Transverse section and the coordinate system used for the analysis of the proposed structure.

the local-normal-mode matching, has been discussed. A comparative study of the performance of the proposed sensor, both in co-propagating and counter-propagating regimes, with that of a corrugated metal garting based sensor has also been carried out in this section. Finally, the concluding remarks have been given in Section IV.

\section{SENSOR StRUCtURE AND MODAl CHARACTERISTICS}

The schematic diagram of the proposed sensor is shown in Fig. 1. It consists of a substrate over which are grown a core of width $a$, an upper cladding of width $b$ and a metal layer having thickness $\Delta$, made of alternate Au-Ag metals along the direction of propagation $z$. The refractive indices of substrate, core, upper cladding and metal regions are denoted by $n_{\mathrm{su}}, n_{\mathrm{co}}, n_{\mathrm{cl}}$ and $n_{m}$, respectively. On the top of the metallic region is a uniform layer of the ambient refractive index (ARI) to be sensed, and unless explicitly mentioned its refractive index $\left(n_{\text {se }}\right)$ has been taken as 1.33. The direction of refractive index variation is taken along the $x$ axis, and the $y$ direction is considered to be extended infinitely. The substrate and the cladding regions are considered to be made of fused Silica and the core region is made of 6.3 $\mathrm{mol} \% \mathrm{GeO}_{2}$ doped $\mathrm{SiO}_{2}$. The wavelength dependence of the refractive indices of various dielectric layers have been obtained by using the Sellmeier relation [17], and that of the metallic regions $\left(n_{m}=\sqrt{\varepsilon(\omega)}\right)$ have been obtained by using the Drude formula [18]

$$
\varepsilon(\omega)=\varepsilon_{\infty}\left[1-\frac{\omega_{p}^{2}}{\omega(\omega+i \Gamma)}\right]
$$

where $\varepsilon_{\infty}$ is the high frequency value of dielectric constant, $\omega_{p}$ is the plasma frequency and $\Gamma$ is the damping frequency, which for $\mathrm{Au}$ and $\mathrm{Ag}$ have been listed in Table I. The dielectric constant at $\lambda=1.55 \mu \mathrm{m}$ as predicted by (1) for $\mathrm{Au}$ and $\mathrm{Ag}$ comes out to be $-96.012+10.956 i$ and $-86.877+8.649 i$, agreeing well to the experimental values $-95.924+10.968 i$ and $-86.642+8.742 i$ reported in [18]. Here it is important to emphasize that the structure will support the SPP, only for the frequencies for which $\operatorname{Re}(\varepsilon(\omega))<-n_{\omega}^{2}$.

In order to carry out a comparative study with the corrugated gratings, in our simulations, the width of the core and upper cladding regions have been taken same as used in [6]. i.e., $a=$ $3 \mu \mathrm{m}$ and $b=4.5 \mu \mathrm{m}$, respectively.

As only TM modes of the planar structure can excite the SPP, we obtained the propagation constant and field distribution for
TABLE I

Metal PARAMETERS USED IN NUMERICAL SimUlations

\begin{tabular}{lcc}
\hline \hline \multicolumn{1}{c}{ Parameter } & Gold & Silver \\
High frequency dielectric constant, $\varepsilon_{\infty}$ & 8.6 & 7.2 \\
Plasma frequency, $\omega_{P}(\mathrm{PHz})$ & 4.264 & 4.414 \\
Damping frequency, $\Gamma(\mathrm{PHz})$ & 0.1274 & 0.1118 \\
\hline
\end{tabular}

both the core mode and the SPP, by solving the well-known TM wave equation [17]

$$
\frac{d}{d x}\left(\frac{1}{n^{2}} \frac{d H_{y}}{d x}\right)+\left(k_{0}^{2}-\frac{\beta^{2}}{n^{2}}\right) H_{y}=0
$$

where, $k_{0}(=2 \pi / \lambda)$ is the free space propagation constant and the $(z, t)$ dependence of the fields is taken of the form $\exp i(\beta z-\omega t), \beta$ being the propagation constant of the mode. The solutions of (2) to obtain the effective indices of the core mode and the SPP has been recalled in Appendix. We first obtain the effective indices $\left(\beta / k_{0}\right)$ of the core mode $\left(n_{\text {core }}\right)$ and the SPP $\left(n_{\mathrm{spp}}\right)$, for different values of $n_{\mathrm{se}}$ and $\Delta$. In Fig. 2(a), (b) we have plotted the variation of the real part of the $n_{\text {core }}$ and $n_{\text {spp }}$ for both the Au and Ag metal coated waveguide sections, with $n_{\text {se }}$, for different values of $\Delta$. Further, their variation with $\Delta$, for $n_{\text {se }}=1.33$, has been plotted in Fig. 3(a), (b). These figures show that the SPP effective index changes much faster as compared to that of the core mode: indicating that SPP field is more sensitive with respect to the change in $n_{\text {se }}$ or $\Delta$, as compared to the core mode.

Having obtained the effective indices and the field distributions of both the modes, the fields are then normalized to carry unit power per unit length along the $y$ direction. The $x$ component of the normalized electric field distribution for both the SPP and the core mode have been plotted in Fig. 4(a), and an amplified curve in the regions close to the metallic layer is shown in Fig. 4(b). These fields have been calculated for Au as the metal layer, at an operating wavelength $\lambda=1.55 \mu \mathrm{m}$ and for $\Delta=10$ $\mathrm{nm}$ and $n_{\mathrm{se}}=1.33$. A similar distribution can also be obtained taking Ag as the metal layer. One can see that the core mode supports a local SPP at the metal/dielectric interface; however the field amplitude is extremely small as compared to that in the waveguide core region. The pure SPP, on the other hand, has its field maxima at the metal/dielectric interfaces and decays sharply in the regions beyond the interfaces, revealing the fact that to increase the modal overlap in the grating region the grating itself should be present in the metallic region.

The grating based sensors involve the measurement of the shift in the resonance wavelength of the transmission spectrum, by measuring the power carried by the core mode at the output end of the optical waveguide. It is, thus, worthwhile to know the loss in the transmitted power due to metal induced core mode loss as compared to the coupling from core mode to the SPP. In Fig. 5 we have plotted the variation of the propagation length 
(a)

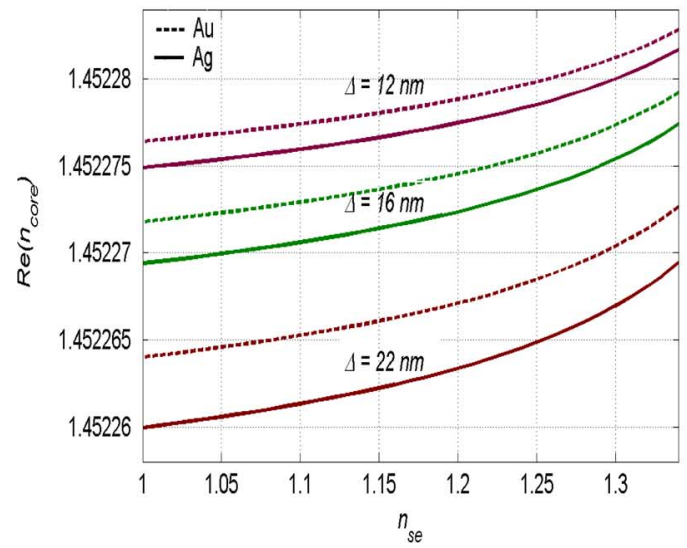

(b)

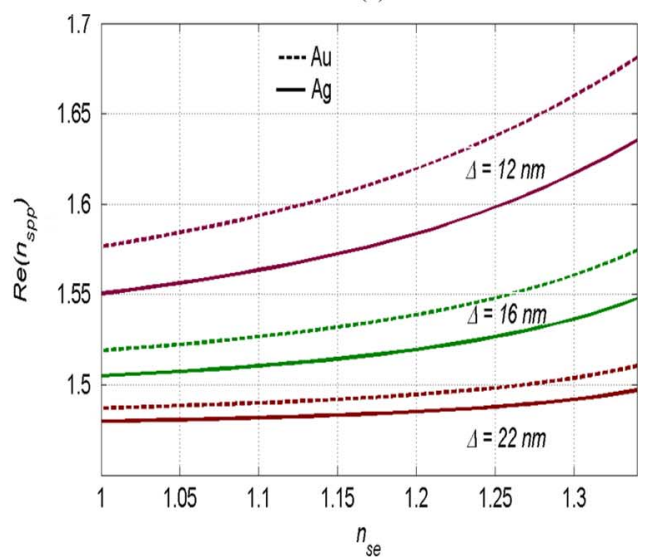

Fig. 2. Variation of the real part of modal effective index with $\mathbf{n}_{\text {se }}$ for $(a)$ core mode and (b) SPP for different values of metal thickness $\Delta$, corresponding to $a=3 \mu \mathrm{m}, b=4.5 \mu \mathrm{m}$, and $\lambda=1.55 \mu \mathrm{m}$.

(a)

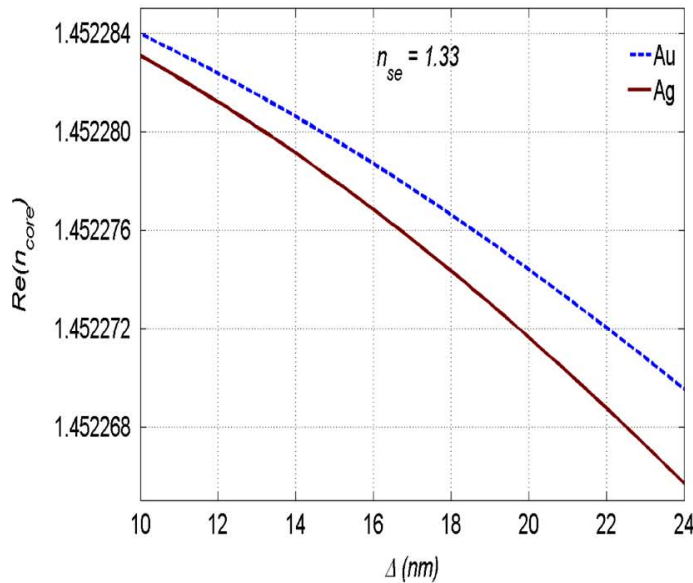

(b)

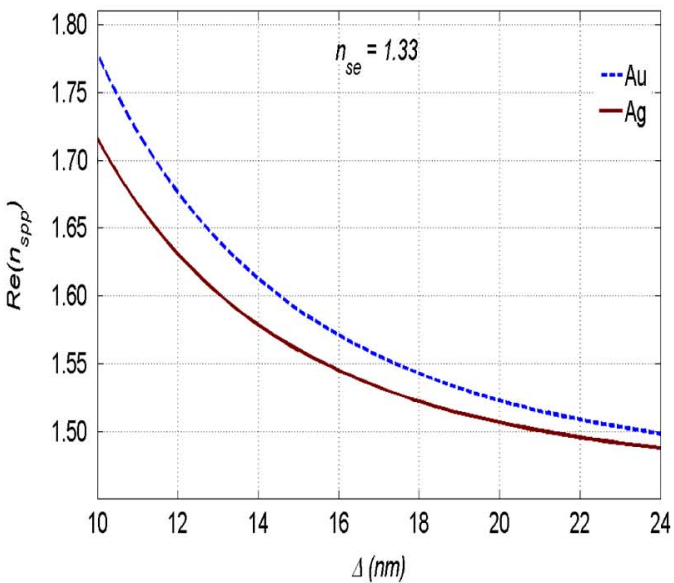

Fig. 3. Variation of the real part of modal effective index with $\Delta$ for $(a)$ core mode and $(b)$ SPP at $n_{\text {se }}=1.33$, corresponding to $a=3 \mu \mathrm{m}, b=4.5 \mu \mathrm{m}$, and $\lambda=1.55 \mu \mathrm{m}$.

$(=(1) /(2 \operatorname{Im}(\beta)))$ of the core mode, for both the Au and $\mathrm{Ag}$ layers, with respect to the metal thickness.

As can be observed, due to a very thick upper cladding the mode loss is very small leading to the propagation length ranging between $4-12 \mathrm{~cm}$. As discussed later, the grating length for the proposed structure comes out to be a few millimeters, which is much smaller compared to the propagation length of the core mode. This means the imaginary part of the $\varepsilon_{m}$ has very little effect on the location of the resonance dip. Further, since for all practical purposes the grating period is decided by the real part of the propagation constant, for the ease of calculation, in the analysis followed we set the damping frequency as zero.

\section{SENSING CHARACTERISTICS}

In order to analyze the mode coupling and sensing characteristics of the proposed structure, we follow the local-normal-mode transfer matrix method [6]. In this method, the grating structure is treated as a sequence of tiny sections of alternate waveguides with uniform $\mathrm{Au}$ and $\mathrm{Ag}$ metal layers. In each of these waveguides the net field is the superposition of all the local-normal-modes with properly chosen amplitudes. The amplitudes of these local modes are calculated by the mode matching, i.e., the continuity of $H_{\mathrm{y}}$ and $E_{\mathrm{x}}$ components, at each junction plane. As a result, at $i$ th junction, the field amplitudes of the $i$ th and $i+1$ th sections are connected by the relations

$$
\begin{aligned}
& \left(A_{f}^{c, i}+A_{b}^{c, i}\right) H_{y}^{c, i}+\left(A_{f}^{P, i}+A_{b}^{P, i}\right) H_{y}^{P, i} \\
& =\left(A_{f}^{c, i+1}+A_{b}^{c, i+1}\right) H_{y}^{c, i+1} \\
& \quad+\left(A_{f}^{P, i+1}+A_{b}^{P, i+1}\right) H_{y}^{P, i+1} \\
& \quad \text { and, } \\
& \left(A_{f}^{c, i}-A_{b}^{c, i}\right) E_{x}^{c, i}+\left(A_{f}^{P, i}-A_{b}^{P, i}\right) E_{x}^{P, i} \\
& =\left(A_{f}^{c, i+1}-A_{b}^{c, i+1}\right) E_{x}^{c, i+1} \\
& \quad+\left(A_{f}^{P, i+1}-A_{b}^{P, i+1}\right) E_{x}^{P, i+1}
\end{aligned}
$$

where, the scripts $c, P, f, b$ stand for core mode, the SPP, forward propagating and backward propagating, respectively, and 
(a)

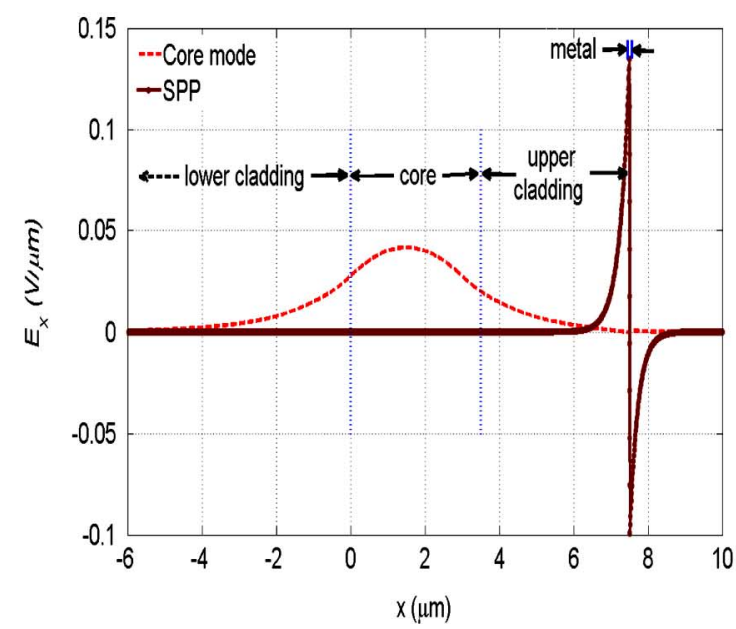

(b)

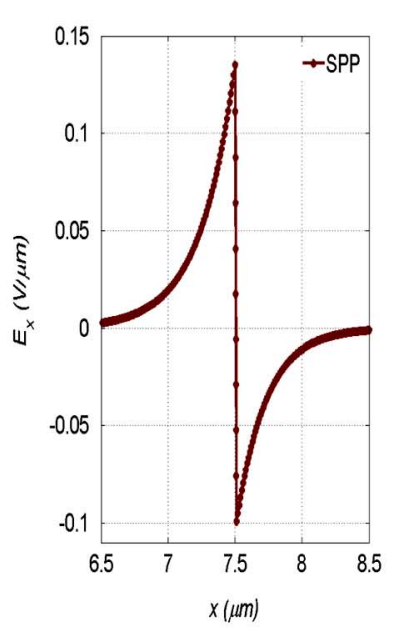

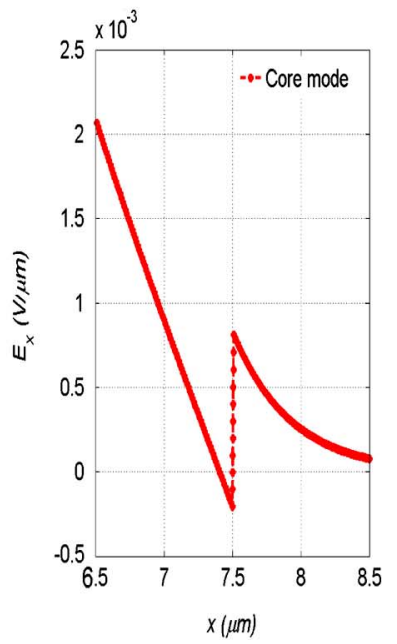

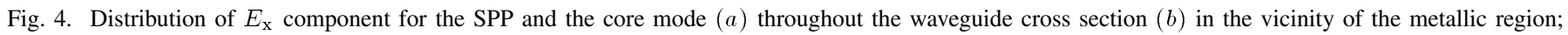
corresponding to $a=3 \mu \mathrm{m}, b=4.5 \mu \mathrm{m}, \Delta=10 \mathrm{~nm}$, and $\lambda=1.55 \mu \mathrm{m}$.

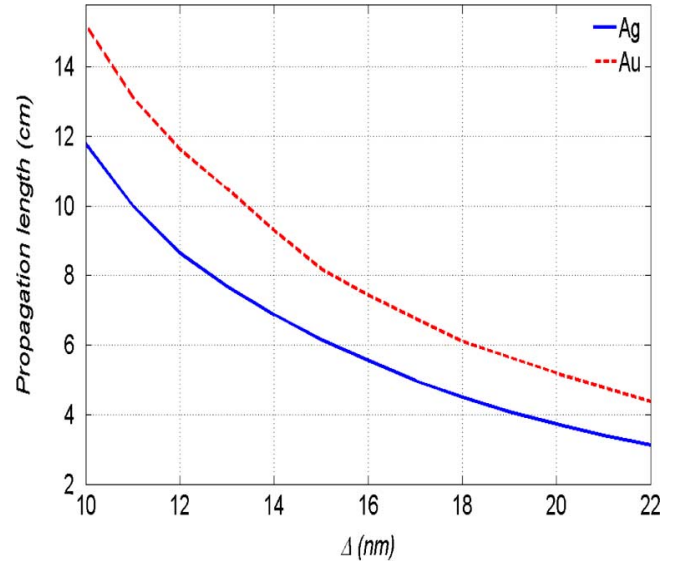

Fig. 5. Propagation length of the core mode, with Au as the metal layer (dotted curve) and $\mathrm{Ag}$ as the metal layer (solid curve,) as a function of $\Delta$ for $n_{\mathrm{se}}=$ $1.33, a=3 \mu \mathrm{m}, b=4.5 \mu \mathrm{m}$, and $\lambda=1.55 \mu \mathrm{m}$.

$A$ represents the field amplitude in the $i$ th or $i+1$ th region. Using the orthonormality of the modes we have

$$
\frac{Z_{0}}{2} n_{e}^{p} \int_{-\infty}^{+\infty} H_{y}^{p, i}(x) \frac{1}{n^{2}(x)} H_{y}^{q, i *}(x) d x=\delta_{p q}
$$

with $Z_{0}=120 \pi \Omega$ being the characteristic impedance of vacuum, $n_{e}^{p}$ being the effective index of $p$ th mode, and $\delta_{p q}$ is the Kronecker symbol.

Using relation, $E_{x}=\left(Z_{0} n_{e}\right) /\left(n^{2}\right) H_{y}$ in (4) and utilizing the (5), after some manipulations we get a matrix $\mathbf{K}^{i+1, i}$ connecting the field amplitudes on either side of the $i / i+1$ junction via the relation

$$
\left(\begin{array}{c}
A_{f}^{c, i+1} \\
A_{b}^{c, i+1} \\
A_{f}^{P, i+1} \\
A_{b}^{P, i+1}
\end{array}\right)=\mathbf{K}^{i+1, i}\left(\begin{array}{c}
A_{f}^{c, i} \\
A_{b}^{c, i} \\
A_{f}^{P, i} \\
A_{b}^{P, i}
\end{array}\right),
$$

with

$$
\mathbf{K}^{i+1, i}=\left(\begin{array}{llll}
k_{f}^{c, c} & k_{b}^{c, c} & k_{f}^{c, P} & k_{b}^{c, P} \\
k_{b}^{c, c} & k_{f}^{c, c} & k_{b}^{c, P} & k_{f}^{c, P} \\
k_{f}^{P, c} & k_{b}^{P, c} & k_{f}^{P, P} & k_{b}^{P, P} \\
k_{b}^{P, c} & k_{b}^{P, c} & k_{b}^{P, P} & k_{f}^{P, P}
\end{array}\right)
$$

where

$$
\begin{aligned}
k_{f}^{m, n}= & \frac{Z_{0}}{4}\left(n_{e}^{m, i+1} \int_{-\infty}^{+\infty} H_{y}^{n, i}(x) \frac{1}{n_{i+1}^{2}(x)} H_{y}^{m, i+1 *}(x) d x\right. \\
& \left.+n_{e}^{n, i} \int_{-\infty}^{+\infty} H_{y}^{n, i}(x) \frac{1}{n_{i}^{2}(x)} H_{y}^{m, i+1 *}(x) d x\right)
\end{aligned}
$$

and

$$
\begin{aligned}
k_{b}^{m, n}= & \frac{Z_{0}}{4}\left(n_{e}^{m, i+1} \int_{-\infty}^{+\infty} H_{y}^{n, i}(x) \frac{1}{n_{i+1}^{2}(x)} H_{y}^{m, i+1 *}(x) d x\right. \\
& \left.-n_{e}^{n, i} \int_{-\infty}^{+\infty} H_{y}^{n, i}(x) \frac{1}{n_{i}^{2}(x)} H_{y}^{m, i+1 *}(x) d x\right)
\end{aligned}
$$

are the various elements of the matrix $\mathbf{K}^{i+1, i}$. The forward and backward propagating modes propagate in the $i+1$ th region with their respective propagation constants. The field amplitudes in the $i+1$ th region at the $i+1 / i+2$ junction can thus be obtained by multiplying the field amplitude matrix by a phase matrix given by (7), shown at the bottom of the following page, where $\Lambda^{i+1}$ is the width of the $i+1$ th region at the $(j+1) /(j+2)$ junction, which is selected from the phase matching condition. Accordingly, $\Lambda^{i}$ for the co-propagating coupling is obtained from the following relation,

$$
\Lambda^{i}\left(\beta_{p}^{i}-\beta_{c}^{i}\right)=\pi
$$


and for the counter-propagating coupling,

$$
\Lambda^{i}\left(\beta_{p}^{i}+\beta_{c}^{i}\right)=\pi
$$

, respectively. Thus, one complete grating period is $\Lambda=\Lambda^{i}+$ $\Lambda^{i+1}$ and the matrix linking the field amplitudes at the beginning and at the end of the period takes the form,

$$
\mathbf{T}_{k}=\mathbf{U}^{i+1} \times \mathbf{K}^{i+1, i} \times \mathbf{U}^{i} \times \mathbf{K}^{i, i-1}
$$

Thus, if the grating contains $N$ repeated sections of period $\Lambda$, the transfer matrix coupling the field amplitudes at the input and output ends of the grating is given by $\mathbf{T}=\prod_{k=1}^{N} \mathbf{T}_{k}$. The transmission and reflection coefficients of the core mode and the SPP through the grating are connected by

$$
\left(\begin{array}{c}
t_{c} \\
0 \\
t_{\mathrm{spp}} \\
0
\end{array}\right)=\mathbf{T}\left(\begin{array}{c}
1 \\
r_{c} \\
0 \\
r_{\mathrm{spp}}
\end{array}\right)
$$

where, $t_{c}$ and $t_{\mathrm{spp}}$ are the transmission coefficients of the core mode and the SPP, respectively, and $r_{c}$ and $r_{\text {spp }}$ are their reflection coefficients, $\mathbf{T}$ is a $4 \times 4$ matrix. From (11) we obtain the reflection and transmission coefficients in terms of the matrix elements as

$$
\begin{aligned}
& r_{c}=\frac{T_{24} T_{41}-T_{21} T_{44}}{T_{22} T_{44}-T_{24} T_{42}} \\
& r_{\mathrm{spp}}=\frac{T_{21} T_{42}-T_{22} T_{41}}{T_{22} T_{44}-T_{24} T_{42}} \\
& \text { and }, \\
& t_{c}= T_{11}+T_{12} r_{c}+T_{14} r_{\mathrm{spp}} .
\end{aligned}
$$

The fractional core mode transmitted power is obtained as

$$
T_{c}=\left|t_{c}\right|^{2} \text {. }
$$

Using (15), in Fig. 6 we have plotted the transmission spectrum of the waveguide for a metal thickness of $\Delta=18 \mathrm{~nm}$, for three different cases namely, a uniform layer of $\mathrm{Au}(-$ curve), an Au-Ag metal grating (solid curve) and a corrugated grating made of $\mathrm{Au}$ with the grating amplitude $1 \mathrm{~nm}$ and a duty cycle $\sim 0.5$ (dashed curve) for $n_{\text {se }}=1.33$. For Au-Ag grating the widths of the $\mathrm{Au}$ and $\mathrm{Ag}$ regions are $\Lambda^{\mathrm{Au}} \sim 11.067 \mu \mathrm{m}$ and $\Lambda^{\mathrm{Ag}} \sim 8.567 \mu \mathrm{m}$, resulting in the grating period $\Lambda \sim$ $19.634 \mu \mathrm{m}$. In order to achieve $30 \%$ transmission at the resonance wavelength $\left(\lambda_{\mathrm{R}}=1.55 \mu \mathrm{m}\right)$, the required grating length comes out to be $L \sim 4.32 \mathrm{~mm}(N=220)$. For the corrugated grating, on the other hand, the required grating length is $L \sim 17.41 \mathrm{~mm}(N=840)$, the periods of two consecutive

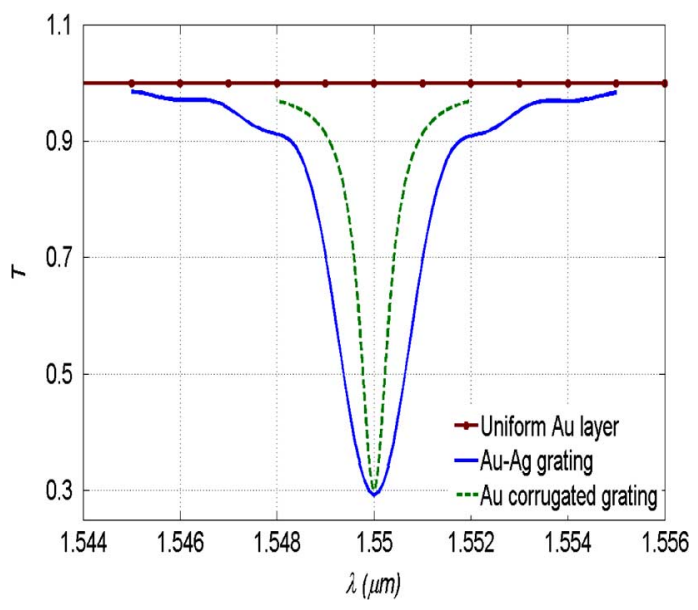

Fig. 6. Transmission spectrum corresponding to a metal thickness of $\Delta=$ $18 \mathrm{~nm}$ with uniform Au layer (—— curve), Au-Ag grating (solid curve) and an Au corrugated grating with grating amplitude of $1 \mathrm{~nm}$. The ARI being water with $n_{\mathrm{se}}=1.33$ and $a=3 \mu \mathrm{m}$, and $b=4.5 \mu \mathrm{m}$.

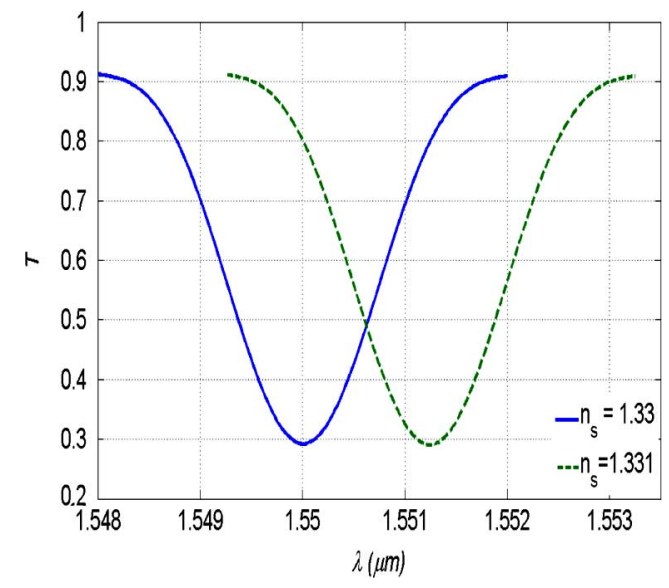

Fig. 7. Transmission spectrum for Au-Ag grating with $\Delta=18 \mathrm{~nm}$, for $n_{\text {se }}=$ 1.33 (solid curve) and $n_{\mathrm{se}}=1.331$ (dashed curve), corresponding to $a=3 \mu$ $\mathrm{m}$, and $b=4.5 \mu \mathrm{m}$.

sections being $\Lambda^{i} \sim 11.067 \mu \mathrm{m}$ and $\Lambda^{i+1} \sim 9.659 \mu \mathrm{m}$ respectively, with grating period $\Lambda \sim 20.726 \mu \mathrm{m}$. It should be noted that in the case of the alternate $\mathrm{Au}-\mathrm{Ag}$ structure the required grating length is reduced to nearly one fourth of that required for a corrugated $\mathrm{Au}$ grating. The transmission bandwidth, however, is relatively large in case of $\mathrm{Au}-\mathrm{Ag}$ grating, which is attributed to the increased modal overlap within the grating region. Next we calculate the spectral shift of the transmission spectrum of Au-Ag grating by changing the ARI $\left(n_{\mathrm{se}}\right)$ from 1.33 to 1.331 , which has been plotted in Fig. 7, showing a spectral shift of $\sim 1242 \mathrm{pm}$, at $\Delta=18 \mathrm{~nm}$. A similar calculation has also been done for corrugated grating. The grating period, grating length

$$
\mathbf{U}^{i+1}=\left(\begin{array}{cccc}
e^{\left(i \beta^{c, i+1} \Lambda^{i+1}\right)} & 0 & 0 & 0 \\
0 & e^{\left(-i \beta^{c, i+1} \Lambda^{i+1}\right)} & 0 & 0 \\
0 & 0 & e^{\left(i \beta^{P, i+1} \Lambda^{i+1}\right)} & 0 \\
0 & 0 & 0 & e^{\left(-i \beta^{P, i+1} \Lambda^{i+1}\right)}
\end{array}\right)
$$


TABLE II

TyPICAL CHARACTERISTICS OF AU-Ag GRATINGS AND CORRUGATED AU GRATING

\begin{tabular}{|c|c|c|c|c|c|c|c|c|}
\hline \multirow[t]{2}{*}{$\begin{array}{c}\text { Metal thickness } \\
\Delta \\
(\mathrm{nm})\end{array}$} & \multicolumn{2}{|c|}{$\begin{array}{l}\text { Grating period for } \mathrm{Au}-\mathrm{Ag} \\
\text { grating } \Lambda(\mu \mathrm{m})\end{array}$} & \multirow[t]{2}{*}{$\begin{array}{l}\text { Grating length } \\
\mathrm{L}(\mathrm{cm})\end{array}$} & \multirow[t]{2}{*}{$\begin{array}{l}\text { Sensitivity } \\
(\mu \mathrm{m} / \mathrm{RIU})\end{array}$} & \multicolumn{2}{|c|}{$\begin{array}{l}\text { Grating period for Corrugated Au } \\
\text { grating } \Lambda(\mu \mathrm{m})\end{array}$} & \multirow[t]{2}{*}{$\begin{array}{l}\text { Grating length } \\
\mathrm{L}(\mathrm{cm})\end{array}$} & \multirow[t]{2}{*}{$\begin{array}{c}\text { Sensitivity } \\
(\mu \mathrm{m} / \mathrm{RIU})\end{array}$} \\
\hline & $\Lambda^{\mathrm{Au}}$ & $\Lambda^{\mathrm{Ag}}$ & & & $\Lambda^{i}$ & $\Lambda^{i+1}$ & & \\
\hline 12 & 4.340 & 3.461 & 0.4681 & 1.087 & 4.340 & 3.594 & 1.1861 & 0.995 \\
\hline 14 & 6.134 & 4.833 & 0.4475 & 1.178 & 6.134 & 5.185 & 1.1489 & 1.210 \\
\hline 16 & 8.367 & 6.527 & 0.4394 & 1.234 & 8.367 & 7.194 & 1.4783 & 1.224 \\
\hline 18 & 11.067 & 8.567 & 0.4319 & 1.242 & 11.067 & 9.658 & 1.7409 & 1.231 \\
\hline 20 & 14.239 & 10.962 & 0.4209 & 1.216 & 14.239 & 12.594 & 1.7576 & 1.217 \\
\hline 22 & 17.865 & 13.706 & 0.4167 & 1.156 & 17.865 & 16.997 & 1.7947 & 1.165 \\
\hline 24 & 21.907 & 16.766 & 0.4177 & 1.102 & 21.907 & 19.837 & 1.9202 & 1.084 \\
\hline
\end{tabular}

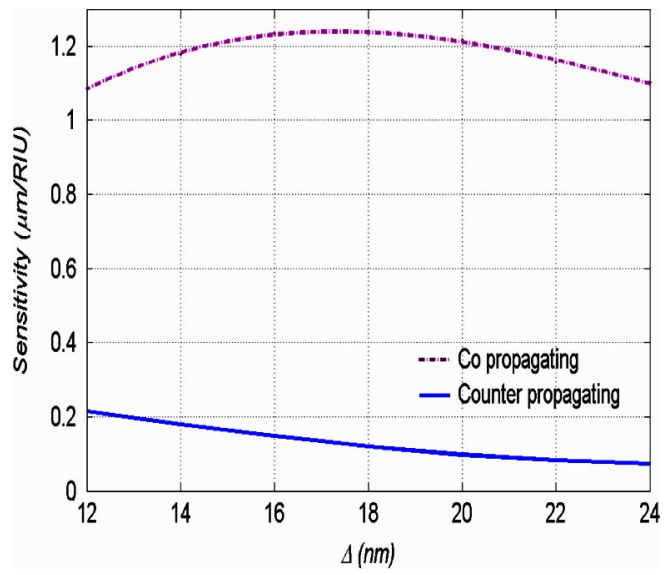

Fig. 8. Sensitivity variation for co propagating coupling (dashed curve) and counter propagating coupling (solid curve), with metal thickness, for an $\mathrm{Au}-\mathrm{Ag}$ grating, with $a=3 \mu \mathrm{m}$, and $b=4.5 \mu \mathrm{m}$.

and the corresponding sensitivity (defined as the spectral shift in the resonance wavelength $\lambda_{\mathrm{R}}$ per unit change in ARI), of both the $\mathrm{Au}-\mathrm{Ag}$ grating and the corrugated Au grating, for different metal thicknesses have been tabulated in Table II. As can be observed, the sensitivity of the $\mathrm{Au}-\mathrm{Ag}$ grating for a given metal thickness is nearly the same (slightly larger) as that of the corrugated Au grating, whereas the required grating length is much shorter for $\mathrm{Au}-\mathrm{Ag}$ gratings, making them a favorable choice over the corrugated gratings.

In Fig. 8 we have shown the variation of sensitivity for both the co-propagating coupling and the counter-propagating coupling, for different metal thicknesses, for the Au-Ag grating. It shows that the structure is more sensitive while operating in co-propagating configuration and also that there exists an optimum metal thickness at which the structure is maximum sensitive to the changes in ARI. For counter-propagating coupling, however, no such optimum metal thickness is obtained.

The existence of the optimum metal thickness as well as the larger sensitivity in the co-propagating configuration can be understood in the following manner [3], [8].

The phase matching conditions for co-propagating (8) and counter-propagating (9) couplings can be written as

$$
\Lambda^{i}=\frac{\lambda_{R}}{2\left(n_{\mathrm{spp}}^{i}-n_{\text {core }}^{i}\right)}
$$

$$
\text { and, } \Lambda^{i}=\frac{\lambda_{R}}{2\left(n_{\mathrm{spp}}^{i}+n_{\mathrm{core}}^{i}\right)} \text {. }
$$

Therefore, in terms of the grating period $(\Lambda)$, the phase matching condition for co-propagating coupling can be written as

$$
\Lambda=\Lambda^{i}+\Lambda^{i+1}=\frac{\lambda_{R}}{2\left(n_{\mathrm{spp}}^{i}-n_{\mathrm{core}}^{i}\right)}+\frac{\lambda_{R}}{2\left(n_{\mathrm{spp}}^{i+1}-n_{\mathrm{core}}^{i+1}\right)}
$$

which gives

$$
\lambda_{R}=2 \Lambda N_{f}
$$

with

$$
N_{f}=\frac{\left(n_{\mathrm{spp}}^{i}-n_{\mathrm{core}}^{i}\right)\left(n_{\mathrm{spp}}^{i+1}-n_{\mathrm{core}}^{i+1}\right)}{\left(n_{\mathrm{spp}}^{i}-n_{\text {core }}^{i}\right)+\left(n_{\mathrm{spp}}^{i+1}-n_{\text {core }}^{i+1}\right)} .
$$

Similarly, for counter-propagating coupling we can write

$$
\begin{aligned}
\Lambda & =\Lambda^{i}+\Lambda^{i+1} \\
& =\frac{\lambda_{R}}{2\left(n_{\mathrm{spp}}^{i}+n_{\mathrm{core}}^{i}\right)}+\frac{\lambda_{R}}{2\left(n_{\mathrm{spp}}^{i+1}+n_{\mathrm{core}}^{i+1}\right)}
\end{aligned}
$$

which gives

$$
\lambda_{R}=2 \Lambda N_{b}
$$

with

$$
N_{b}=\frac{\left(n_{\mathrm{spp}}^{i}+n_{\text {core }}^{i}\right)\left(n_{\mathrm{spp}}^{i+1}+n_{\text {core }}^{i+1}\right)}{\left(n_{\mathrm{spp}}^{i}+n_{\text {core }}^{i}\right)+\left(n_{\mathrm{spp}}^{i+1}+n_{\text {core }}^{i+1}\right)} .
$$

The sensitivity (fractional change in $\lambda_{R}$ due to a given change in $n_{\text {se }}$ ) is, thus, basically proportional to $\delta N_{f} / N_{f}$, for co-propagating coupling and proportional to $\delta N_{b} / N_{b}$, for counter-propagating coupling. Further, it is also clear from these expressions that for a given $\Delta$, the sensitivity of the co-propagating modes based sensor will be $\delta N_{f} N_{b} / \delta N_{b} N_{f}$ times larger than that of a counter-propagating modes based device explaining its higher sensitivity. In order to understand the dependence of the sensitivity on $\Delta$ in the two cases, we have plotted, in Fig. 9, the variation of $\delta N_{f, b} / N_{f, b}$ with $\Delta$. It is clear from this figure that the parameter $\delta N_{f} / N_{f}$ first increases with increasing the metal thickness and after attaining a certain maximum value, near a metal thickness of $18 \mathrm{~nm}$, starts decreasing with further increase 


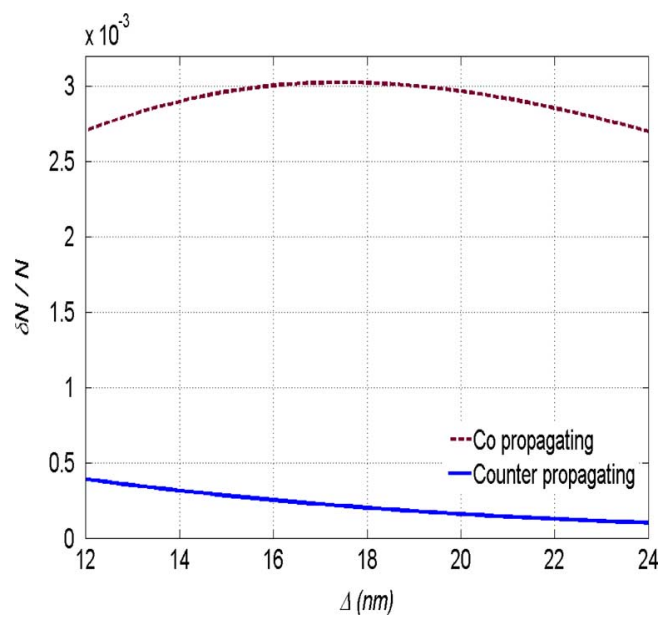

Fig. 9. Variation of $\delta N_{f, b} / N_{f, b}$ with $\Delta$ for co propagating (dashed curve) and counter propagating (solid curve) coupling, for $a=3 \mu \mathrm{m}, b=4.5 \mu \mathrm{m}$, and $\lambda=1.55 \mu \mathrm{m}$.

in $\Delta$; explaining the existence of the optimum metal thickness $\Delta_{\text {opt }}$ for the co-propagating modes based sensor. On the other hand the parameter $\delta N_{b} / N_{b}$ keeps on decreasing monotonically with the increase in $\Delta$, explaining the absence of any optimum value of $\Delta$ for the counter propagating modes based sensors.

Using the peak/dip finding algorithm [19], and assuming a detector with a resolution of $1 \mathrm{pm}$, changes as small as $8.052 \times$ $10^{-7}$ in the ARI can be detected at the optimum metal thickness $(\sim 17 \mathrm{~nm})$. The present grating structure not only requires much smaller grating length but also has comparable sensitivity with those reported for ultra thin cladding, pure SPP based photosensitive gratings [1]-[8], and larger sensitivity as compared to the gratings in highly miniaturized high index contrast $\mathrm{Si} / \mathrm{SiO}_{2}$ waveguides [14].

\section{CONCLUSION}

An exact coupled mode theory is used to study a novel highly sensitive, miniaturized, integrated optic refractive index sensor based on the Au-Ag surface gratings. The grating is considered to be made of alternate layers of equi-thick $\mathrm{Au}$ and $\mathrm{Ag}$ regions along the direction of propagation, on the surface of the waveguide. It has been shown that the present structure requires much shorter grating lengths as compared to the corrugated Au gratings with same metal thickness and nearly the same sensitivity. Further, both the co-propagating and counter-propagating gratings are studied. It has been found that for co-propagating modes the structure is maximum sensitive at an optimum metal thickness, however, such an optimum metal thickness does not exist for counter-propagating modes. However, it should be noted that the transmission bandwidth of an Au-Ag grating is relatively large in comparison with the transmission bandwidth of the $\mathrm{Au}$ corrugated grating.

\section{APPENDIX}

Here we give the field expressions corresponding to the solution of (2) to obtain the propagation constant for the core mode and the SPP. Since for the core mode the field is guided within the core region and decays in all regions except the core, and for the SPP the field decays in all the regions, the solution of (2) for both the core mode and the SPP can be written as [4], [17]

$$
\begin{aligned}
H_{y}= & A_{\mathrm{su}} \exp \left(\gamma_{\mathrm{su}} x\right) \quad \text { for } x<0 \\
= & A_{\mathrm{co}} \exp \left(\gamma_{\mathrm{co}} x\right) \\
& +B_{\mathrm{co}} \exp \left(-\gamma_{\mathrm{co}} x\right) \text { for } 0<x<a \\
= & A_{\mathrm{cl}} \exp \left(\gamma_{\mathrm{cl}}(x-a)\right) \\
& +B_{\mathrm{cl}} \exp \left(-\gamma_{\mathrm{cl}}(x-a)\right) \text { for } a<x<b \\
= & A_{m} \exp \left(\gamma_{m}(x-b)\right) \\
& +B_{m} \exp \left(-\gamma_{m}(x-b)\right) \text { for } b<x<b+\Delta \\
= & B_{\mathrm{se}} \exp \left(-\gamma_{\mathrm{se}} x\right) \text { for } x>b+\Delta
\end{aligned}
$$

where, su, co, cl, $m$, se. stand for substrate, core, cladding, metal and sensing regions, respectively, and $\gamma_{t}$ is defined as $\gamma_{t}=i \kappa_{t}=\sqrt{k_{0}^{2}\left(n_{t}^{2}-n_{e}^{2}\right)}$ with $t=\mathrm{su}, \mathrm{co}, \mathrm{cl}, m, \mathrm{se}$, with $n_{\mathrm{cl}}<n_{e}<n_{\mathrm{co}}$ for the core mode and $n_{e}>n_{\mathrm{co}}$ for the SPP.

The other two nonzero field components, $E_{\mathrm{x}}$ and $E_{\mathrm{z}}$ are related to the $H_{\mathrm{y}}$ component via the relation [14]

$$
E_{x}=\frac{Z_{0} n_{e}}{n^{2}} H_{y} \text { and } E_{z}=-i \frac{Z_{0} n_{e}}{n^{2}} \frac{d H_{y}}{d x}
$$

where $Z_{0}$ is the characteristics impedance of the free space. In order to obtain the propagation constant we apply the boundary condition that $H_{\mathrm{y}}$ and $E_{\mathrm{z}}$ must be continuous at every dielectric discontinuity, $x=a, b, b+\Delta$. Following this an eigen value equation of the type $f(\beta)=0$ is obtained, here $\beta$ is the propagation constant of the mode.

Now, to obtain the complex value of the propagation constant we plot a function

$$
F=\left|\frac{1}{f(\beta)}\right|^{2}
$$

against the trial values of effective index $n_{\mathrm{e}}\left(=\beta / k_{0}\right.$ with $n_{\mathrm{cl}}<$ $n_{\mathrm{e}}<n_{\mathrm{c}}$ for the core mode and $n_{\mathrm{e}}>n_{\mathrm{c}}$ for the SPP) and, a Lorentzian curve is obtained [20]. The value of $n_{\mathrm{e}}$ at which the Lorentzian has its peak gives the real value of the effective index and the FWHM gives the imaginary value of the effective index of the mode.

Having obtained the propagation constant and the field distributions in various regions, the fields are then normalized to carry unit power along the $y$ direction using the relation,

$$
\frac{N^{2} Z_{0} n_{e}}{2} \int_{-\infty}^{\infty} \frac{1}{n^{2}}\left|H_{y}\right|^{2} d x=1
$$

where, $N$ is the normalization factor. The propagation constants and the normalized fields are thus obtained in our analysis.

\section{REFERENCES}

[1] G. Nemova and R. Kashyap, "Fiber-bragg-grating-assisted surface plasmon-polariton sensor," Opt. Lett., vol. 31, pp. 2118-2120, 2006.

[2] G. Nemova and R. Kashyap, "Modeling of plasmon-polariton refractive-index hollow core sensors assisted by a fiber bragg grating," $J$. Lightw. Technol., vol. 24, no. 10, pp. 3789-3796, Oct. 2006.

[3] G. Nemova and R. Kashyap, "Theoretical model of a planar integrated refractive index sensor based on surface plasmon-polariton excitation with a long period grating," J. Opt. Soc. Amer. B vol. 24, pp. 2696-2701, 2007. 
[4] G. Nemova and R. Kashyap, "Theoretical model of a planar integrated refractive index sensor based on surface plasmon-polariton excitation," Opt. Commun., vol. 275, pp. 76-82, 2007.

[5] G. Nemova and R. Kashyap, "A compact integrated planar-waveguide refractive-index sensor based on a corrugated metal grating," J. Lightw. Technol., vol. 25, no. 8, pp. 2244-2250, Aug. 2007.

[6] Y.-J. He, Y.-L. Lo, and J.-F. Huang, "Optical-fiber surface-plasmonresonance sensor employing long-period fiber gratings in multiplexing," J. Opt. Soc. Amer. B., vol. 23, pp. 801-811, 2006.

[7] Y. Y. Shevchenko and J. Albert, "Plasmon resonances in gold-coated tilted fiber bragg gratings," Opt. Lett., vol. 32, pp. 211-213, 2007.

[8] S. M. Tripathi, A. Kumar, E. Marin, and J.-P. Meunier, "Side-polished optical fiber grating-based refractive index sensors utilizing the pure surface plasmon polariton," J. Lightw. Technol., vol. 26, no. 13, pp. 1980-1985, Jul. 2008.

[9] S. M. Tripathi, A. Kumar, E. Marin, and J.-P. Meunier, "Refractive index sensing characteristics of dual resonance long period gratings in bare and metal-coated D-shaped fibers," Appl. Opt., vol. 48, pp. G53-G58, 2009.

[10] V. R. Almeida, Q. Xu, C. A. Barrios, and M. Lipson, "Guiding and confining light in void nanostructure," Opt. Lett., vol. 29, pp. 1209-1211, 2004.

[11] Q. Xu, V. R. Almeida, R. R. Panepucci, and M. Lipson, "Experimental demonstration of guiding and confining light in nanometer-size lowrefractive-index material," Opt. Lett., vol. 29, pp. 1626-1628, 2004.

[12] "Lab on a Chip," in Nature (Insight Supplement) pp. 367-418, vol. 442, 2006.

[13] K. K. Lee, D. R. Lim, and L. C. Kimerling, "Fabrication of ultralowloss $\mathrm{Si} / \mathrm{SiO}_{2}$ waveguides by roughness reduction," Opt. Lett., vol. 26, pp. 1888-1890, 2001

[14] S. M. Tripathi, A. Kumar, E. Marin, and J.-P. Meunier, "Bragg grating based biochemical sensor using submicron $\mathrm{Si} / \mathrm{SiO}_{2}$ waveguides for lab-on-a-chip applications: A novel design," Appl. Opt., vol. 48, pp. 4562-4567, 2009.

[15] Y.-C. Lu and W.-P. Huang, "Influence of mode loss on the feasibility of grating-assisted optical fiber surface plasmon resonance refractive index sensors," J. Lightw. Technol., vol. 27, no. 21, pp. 4804-4808, Nov. 2009.

[16] H. Nishihara, M. Haruna, and T. Suhara, Optical Integrated Circuits. New York: McGraw-Hill, 1985.

[17] A. K. Ghatak and K. Thyagarajan, Introduction to Fiber Optics. Cambridge, U.K.: Cambridge Univ. Press, 1998, ch. 6, pp. 82-83.

[18] D. W. Lynch and W. R. Hunter, "Comments on the optical constants of metals and an introduction to the data for several metals," in Handbook of Optical Constants of Solids, E. D. Palik, Ed. San Diego, CA: Academic, 1985, vol. 1, pt. II, pp. 275-368.

[19] User's manual, HP 71450B/1B/1/2B Optical Spectrum Analyzer.

[20] M. R. Ramadas, E. Garmire, A. K. Ghatak, K. Thyagarajan, and M. R. Shenoy, "Analysis of absorbing and leaky planar waveguides: A novel method," Opt. Lett., vol. 14, pp. 376-378, 1989.

Saurabh Mani Tripathi was born in Gorakhpur, India, on May 29, 1982. He received the B.Sc. and M.Sc. degrees from Gorakhpur University, India, in 2003 and 2005, respectively. He is currently pursuing the Ph.D. degree with the Department of Physics, Indian Institute of Technology, New Delhi.

His research interests are modal interference based guided wave devices, integrated optical devices, optical waveguide gratings and surface plasmon polariton based sensors and devices.
Arun Kumar received the M.Sc. and Ph.D. degrees in physics from the Indian Institute of Technology, New Delhi, India, in 1972 and 1976, respectively.

Since 1977, he has been a member of the faculty of the Physics Department, Indian Institute of Technology, New Delhi, where he has been a Professor since 1995 . He was a Visiting Scientist with the Technical University of Hamburg-Harburg, Germany, as a Humboldt Research Fellow in 1980-1981; the Opto-electronic Group, Strathclyde University, Glasgow, U.K., in 1988; the National Institute of Standards and Technology, Boulder, CO, in 1993 and 1994; the University of Nice, France, in 1996; and the University of Jean Monnet, Saint-Etienne, France in 1999, 2004, 2006 and 2007. He has authored or coauthored more than 90 research papers in the international journals. His research interests are in the field of optical waveguides, fiber optic sensors, and polarization mode dispersion.

Prof. Kumar is a member of the Optical Society of America.

Emmanuel Marin was born in Valence, France, on February 24, 1970. He received the Ph.D. degree from the University Jean Monnet, Saint-Etienne, France, in 2000.

His doctoral work focused on the prediction of Bragg grating effects in optical fiber components. After postdoctoral work on phononic properties of microstructured optical fibers at Bath University, Bath, U.K., he has been with the Department of Physics, University Jean Monnet, as an Assistant Professor since 2001. His current research activities include fiber-optic sensors and waveguides.

Jean-Pierre Meunier (M'01) graduated from the University of Lyon I, France, in 1971. He received the Diplôme d'Etudes Approfondies degree in quantum mechanics and the Doctorat de Spécialité degree in the theoretical nuclear physics from the Nuclear Physics Institute of Lyon, France, in 1972 and 1973, respectively, and the Doctorat d'Etat ès Science degree in physics from the University Jean Monnet, Saint-Etienne, France, in 1986.

Sience 1974, he has been with the department of Physics, University Jean Monnet, where he is currently a Professor working in the signal and electromagnetic theory, integrated and guided wave optics, and applied mathematics. In 1976, he cofounded a laboratory and transferred to work on optical waveguides. His current research interests are in developing theoretical tools for the modeling and characterization of passive and active optical guided wave devices. He has authored or coauthored more than 135 research papers in the international journals and conference proceedings. He is editor of two books on optical fibers for telecommunications.

Prof. Meunier is a member of the French Optical Society, the Optical Society of America, and IEEE LEOS. 